\title{
A Qualitative Study of Young Adults Experience with a Reminiscence-Based Therapy for Depressive Symptoms
}

\author{
Hallford, D. J. ${ }^{1,2}$, Mellor, D. ${ }^{1}, \&$ Burgat, M. E. ${ }^{1}$ \\ ${ }^{1}$ School of Psychology, Deakin University, 1 Gherinhap St, Geelong, Victoria 3220, \\ Australia \\ ${ }^{2}$ Corresponding Author, School of Psychology, Deakin University, 221 Burwood Hwy, \\ Burwood, Victoria 3125, Melbourne, Australia, Phone: + 6139251 7777, Email: \\ david.hallford@deakin.edu.au
}

Please cite as:

Hallford, D. J., Mellor, D., \& Burgat, M. E. (2019). A qualitative study of young adults experience with a reminiscence-based therapy for depressive symptoms. Emerging Adulthood, 7(4), 279-290. https://doi.org/10.1177/2167696818769175 


\begin{abstract}
Reminiscence therapy is a novel psychosocial approach to treating depressive symptoms in young adults. We present the first qualitative inquiry into young adults' experience of reminiscence-based therapy, aimed at exploring attitudes towards this approach and their view of processes underpinning positive change. Help-seeking young adults (mean age $20.8, S D=1.6$ ) with at least moderate depressive symptoms were interviewed following a course of cognitive-reminiscence therapy. The data were analysed using thematic analysis. The intervention was reported to be a positive experience which led to positive outcomes. Change was attributed to common therapy factors, and specific factors of cognitive reframing, increasing awareness of a broader life story, learning from the past to feel more confident and able to cope, and strengthened self-worth and self-acceptance. These findings provide unique insight into young adults' experience with reminiscencebased treatment for depressive symptoms, and indicates it is generally acceptable, useful, and age-appropriate for them.
\end{abstract}

Keywords: reminiscence therapy, depression, cognitive-reminiscence therapy, depression, young adults, qualitative 
Reminiscence-based therapies are an effective psychotherapeutic approach for the treatment of depression symptoms in older adults, with recent meta-analytic evidence showing a large and homogenous effect size of $g=1.09$ across 23 trials (Pinquart \& Forstmeier, 2012). Cognitive-reminiscence therapy (CRT; Cappeliez, 2002) has emerged as a promising framework in which to use guided reminiscence to reduce depressive symptoms. CRT adopts cognitive therapy (Beck, Rush, Shaw, \& Emery, 1979) and stress and coping (Billings \& Moos, 1982) conceptualizations of depressive psychopathology, and involves a therapist-assisted review of past experiences. Therapists pragmatically use various therapeutic techniques to help people review these experiences more adaptively, and in relation to their current self-concept and life narratives. In this sense, CRT is an integrative psychotherapy, whereby the retrieval and guided processing of previous life experiences is used as a vehicle for the application of these therapeutic techniques. Through this process, individuals can learn more adaptive ways of construing experiences. These include cognitive restructuring of previous negative events, analysing past coping to recognize achievements and how these were attained, identifying and crystallizing existential meaning in one's life, and developing cohesive and adaptive narratives about one's life and self (Watt \& Cappeliez, 1995, 2000).

CRT was initially developed for use with older adults (Watt \& Cappeliez, 1995) and has been studied exclusively in this age demographic (Cappeliez, 2002, Karimi et al., 2010; Shellman, Mokel \& Hewitt, 2009; Watt \& Cappeliez, 2000). This is unsurprising given that reminiscence has typically been conceptualized as the domain of older adults. More recently, removed for blinding have proposed that these interventions may be appropriate and useful for the treatment of depression in young adults. The prevalence of 
clinical depression has been found to be relatively higher for young adults than other age groups (e.g. Hasin, Goodwin, Stinson, \& Grant, 2005; Rohde, Lewinsohn, Klein, Seeley, Gau, 2013; Slade et al., 2009). The onset of clinical depression in early adulthood is associated with relatively more severe clinical symptomatology, prolonged disease course, and poorer outcomes compared to depression in other age-groups (Coryell et al., 2009; Korten, Comijs, Lamers, Penninx, 2012; Zisook et al., 2007). Effective intervention for depression therefore appears particularly important during this vulnerable period so as to curtail the chronicity and morbidity of symptoms. Further, to ensure that adaptive psychosocial development can be achieved and a stable and positive sense of identify formed and maintained, and to reduce the likelihood of future occurrences of depression. Young adulthood marks a significant period of psychosocial development in life (Arnett, 2004), during which individuals forge their self-concept in the context of an emerging narrative identity (Habermas \& Bluck, 2000), and strive to achieve markers of adulthood such as increased autonomy and progress in occupational and educational domains. Congruent with this, young adults, relative to older adults, appear to draw more frequently on their autobiographical memories for purposes of self-concept clarity, selfcontinuity, and self-direction for future goals or task-based behaviour (Bluck \& Alea, $2008,2009)$. However, depressed youth identify how adversity in this life stage can derail their narratives of meeting normative expectations, and contribute to their depression (Issakainen \& Hänninen, 2016). Given that CRT aims to review and integrate individuals' past experiences to assist in these adaptive processes, it appears to also be suited to the psychosocial needs of young adults, for whom few treatments have been specifically validated (Purcell et al., 2013). 
Against this background, we recently implemented a randomized controlled trial to assess the potential of CRT to reduce depressive symptoms in young adults (blinded for review). Fourteen young adults received CRT, with large reductions observed in depressive symptoms from pre- to post-intervention (Cohen's $d=1.59$ ), which were maintained at the three month follow-up $(d=1.51)$. In the comparison group no significant effect over time was found. Based on Jacobson and Traux's (1991) reliable change index, at post-intervention $86 \%$ of the participants in the CRT group improved, $7 \%$ showed no change, and 7\% deteriorated. At the three month follow-up, $77 \%$ had improved and 23\% showed no reliable change. Large effects $(d>.80)$ were also reported on secondary outcomes of self-esteem, self-efficacy, meaning in life, and optimism, which were also maintained at three-month follow-up with the exception of optimism which had a moderate-to-large effect $(d=.74)$.

These findings are consistent with Watt and Cappeliez's (1995) proposition that the effects of guided reminiscence on depressive symptoms are likely to occur indirectly through improvements in self-concept and well-being in domains such as self-esteem, self-confidence, and meaning in life. Other empirical investigations into the processes underpinning the effect of reminiscence-based therapies on depressive symptoms have begun to emerge, and have supported this theory (e.g.; Korte, Westerhof, \& Bohlmeijer, 2012; Westerhof, Bohlmeijer, van Beljouw, \& Pot, 2010). However, these quantitative studies have focused on older adult populations, as have four qualitative studies of this nature (Binder et al., 2009; Malette \& Oliver, 2006; McDougall, Blixen \& Suen, 1997; Xiao, Kwong, Pang, \& Mok, 2012). Overall, the findings from these studies indicate theory-consistent processes across reminiscence-based therapies that contribute to 
positive outcomes, including identification of meaning in life, a focus on strengths and positive experiences, integration and continuity of experiences, and reflecting on and reconciling negatively-valenced memories. However, only one of these qualitative studies (McDougall et al., 1997) actually involved participants that were help-seeking for depressive symptoms.

Although preliminary quantitative outcomes show positive change (removed for blinding) and are consistent with theories of change, young adults' attitudes towards reminiscence-based therapy and its acceptability are not yet known. Given the differing psychosocial goals (Arnett, 2004) and more future-oriented time perspective that younger adults possess relative to older adults (Carstensen, 2006), it is possible that they have unique experiences in reminiscence-based therapy relative to older adults, and may have different attitudes towards it. Young adults' perceptions of how change occurs in CRT might also differ from older adults, and it is unknown how congruent these are with theoretical postulations and empirical evidence to date. Qualitative research into the experience of young adults who engage in this therapy may provide nuanced insights into its acceptability, and the processes of the intervention they perceive to be related to positive change. To address this research gap, the current study presents an analysis of interviews conducted with participants from the aforementioned clinical trial of CRT for depressive symptoms in young adults. The overarching aim of this study was to use an open-ended interviewing approach to elicit from the young adult clients their experience of this type of psychotherapeutic intervention. More specifically, the study focused on their attitudes towards reminiscence-based therapy and structural components of CRT (e.g., length, homework etc.), information about what they found particularly helpful or 
not unhelpful, and whether they could identify any processes through which change

occurred. Prior to this, we present a description of CRT structure and processes.

\section{Cognitive-Reminiscence Therapy for Young Adults}

The basic format and content of the cognitive-reminiscence intervention for young adults was adapted from a manual developed and evaluated by Watt and Cappeliez $(1995,2000)$ in their work with older adults. Several changes were made: Separate protocols for integrative reminiscence (reminiscence with the function of identification of continuity and meaning across experiences, and reconciliation of past negative events) and instrumental reminiscence therapy (the recollection of past coping and problemsolving experiences to enhance self-efficacy and perceived control) were combined so that both forms of reminiscence were engaged in within each session (Cappeliez, 2002); therapy was individual rather than group-based; and adaptations were made to questions used in sessions and homework sheets to make them more age-appropriate. This manual is available from the corresponding author.

In CRT, six, 60-minute weekly sessions are provided. In the first session, current depression-related issues are briefly reviewed and psychoeducation is then provided on: cognitive therapy and stress and coping models of depression, integrative and instrumental types of reminiscence, and the structure and process of CRT. A problem list is generated for use in subsequent sessions. Memories related to the first topic of turning points (times of significant change in one's life) are elicited, and several are nominated as relevant to integrative reminiscence and instrumental reminiscence. These memories are elaborated on and sufficient details are elicited to inform therapeutic work. Cognitive therapy techniques (Beck et al., 1979) are used while engaging in integrative-type 
reminiscence to promote cognitive distancing and reattribution, to emphasise positive and more balanced evaluations of experiences, to reinterpret negative memories to be more rational and adaptive, to appraise self-worth and meaning in life in accordance with internal standards, and to foster a sense of continuity and a positive narrative identity. On engaging in instrumental-type reminiscence, a stress and coping framework (Billings \& Moos, 1982) is used to conceptualise past experiences and promote the appraisal of stressors as challenges rather than threats, recognise and develop coping resources, and promote awareness of prior constructive problem-focussed coping. A summary of the reminiscence work is then provided and feedback is elicited on the individual's impressions, what they have learned, and any changes in thinking that occurred. Homework sheets containing information about problem-solving, and questions designed to elicit memories related to the next week's topic are provided. The remaining sessions follow a procedure of reviewing homework, identifying memories to elaborate on and engaging in therapeutic work with these memories, reflecting on this reminiscence, and then utilising a problem-solving framework to choose solutions to identified problems to implement over the next week. In the last session the therapist provides an overview of the work completed over the course of the intervention, and the participant is invited to reflect on their experience. A different topic is used each week for therapeutic reminiscence work: turning points, family life, significant/important activities in life, loves and hates, stressful experiences, and meaning in life. A more thorough discussion of the intervention can be found in Watt and Cappeliez (2000).

\section{Methods}

\section{Participants}


The sample comprised 13 young adults who were recruited in 2013-2015 from a community youth mental health service in Melbourne, Australia as part of a clinical trial of CRT. In Mental health clinicians working at the youth mental health service identified help-seeking youth who were eligible to participate in the study through assessments conducted as part of their normal intake protocol, and their permission was sought for a researcher to follow-up with them and discuss the study and obtain consent to participate. All 14 participants who consecutively entered and finished a course of CRT were invited to engage in an interview. One participant was excluded after three failed attempts to conduct the interview. Inclusion criteria were: 18 or more years of age and at least moderate depressive symptoms (as indicated by a score of seven or higher on the depression subscale of the Depression, Anxiety and Stress Scales-21, a reliable and valid assessment of depressive symptoms (Lovibond \& Lovibond, 1995). Exclusion criteria were: concurrent treatment for depression; physical causes for depressive symptoms; high risk of suicide or harm to others; and a clinically-significant or diagnosable mental disorder related to psychotic symptoms, disordered eating, manic symptoms, body image disturbance, anxiety, substance use, or sexual dysfunction that would trump depressive symptoms as the primary presenting problem. Of these 13 participants, seven were female and six were male. Their ages ranged from 18 to 23 years, with a mean of 20.8 $(S D=1.6)$. Regarding highest educational attainment, one participant had completed postgraduate study, three an undergraduate degree or diploma, eight high school, and one primary school. Seven (53.8\%) were currently students. Eight (61.5\%) identified as being in a romantic relationship (none married). Mean baseline levels of depressive symptoms were $12.1(S D=3.4$, range $0-21)$, indicating an elevation in the "severe" range relative 
to the Australian adult population (Lovibond \& Lovibond, 1995). Six participants had received previous treatment for issues related to mental health: Three had seen a psychologist, two had seen a school counsellor, and one had received both antidepressant medication and psychotherapy.

\section{Materials}

A semi-structured interview schedule was used. The guiding interview questions were:

1. In general, how did you find your experience receiving the intervention?

2. In general, how did you find the overall approach?

3. How did you find the experience of talking over past events in your life?

4. Were there any aspects of the intervention that you found particularly beneficial or helpful?

5. Were there any aspects of the intervention that you found particularly unhelpful or difficult?

6. How did you find completing the worksheets between sessions?

7. What did you think about the length of the intervention?

8. What would you like to have seen included that wasn't?

9. Has anything changed for you since you started the intervention?

10. Have you experienced any changes in how you cope with adversity/understand yourself and your life?

These questions were designed to be open-ended in nature in order to elicit reflection on the experience of receiving the intervention. The interviewer followed-up 
these questions with others to probe and explore further the participants' experience of the intervention, and the means through which any change occurred. In particular, when interviewees reported change as a result of CRT, the interviewer explored their beliefs about how these changes occurred for them. For example, "How was the intervention helpful in creating that change?", "Can you elaborate on what you mean by that", "What happened in the intervention that might have led to that?".

\section{Procedure}

Prior to data collection ethics approval to conduct the study was obtained from the university ethics committee. Participants received six weekly, individual sessions of CRT. The therapist was the first author, a supervised provisionally-registered psychologist undertaking a doctoral degree in clinical psychology. Results from a fidelity checklist indicated $100 \%$ adherence for the specified CRT components, except for brief structured problem-solving which was used in $85.7 \%$ of the required sessions due to time constraints.

To partly mitigate against a response bias, the interviews were conducted by the third author, who was not involved in any other aspect of the clinical trial. The interviewer was briefed on the content and process of cognitive-reminiscence therapy, and the purpose of the interview questions. The provision of such information was deemed necessary to establish a context for the interviews and relevant follow-up probing questions. The interviewer attempted to retain a curious and exploratory approach to interviewing, with the aim of drawing out interviewees' own perceptions of the intervention approach and how any change occurred. Interviews were reviewed by the first author as they were completed, and an ongoing dialogue was preserved with the 
interviewer to further refine the interview technique in line with the aims of the study.

The interviews took place between one day and one week of participants' last session of therapy, with the exception of one interview which occurred two weeks afterwards.

Interviews were conducted over the phone, a valid and effective medium for conducting semi-structured interviews of sensitive topics (Cachia \& Millward, 2011; Novick, 2008), and recorded on a digital voice recording device (mean length $=27$ minutes and four seconds). Participants were not provided with any specific incentive to participate.

\section{Data Analysis}

Thematic analysis was conducted as outlined by Braun and Clarke (2006). Thematic analysis is an atheoretical approach to identifying, analysing, and reporting patterns within qualitative data sets, and is commonly used in the study of psychotherapy (e.g. van Rooij, Zinn, Shoenmakers, \& Mheen, 2012; May, Strauss, Coyle \& Hayward, 2012). Thematic analysis involves an iterative process through which data are coded and then grouped into overarching themes that are interpreted for their meaning. Six phases are passed through: becoming familiar with the data, generating initial codes, searching for themes, reviewing themes, defining and naming themes, and producing a report. A primarily semantic approach was used to analyse the surface meaning of the entire data set to identify codes and then themes relevant to the research questions. An essentialist epistemology was adopted, that is, the personal motivations, meanings, and experiences that were expressed by participants through language were assumed to reflect reality. An inductive approach to analysis was used, however, this was likely to be implicitly guided during the initial coding phase by themes identified in previous qualitative research on 
reminiscence-based therapies (see above). In this context the analysts strived to retain openness to themes expressed by participants, in light of the novel population.

Analysis was conducted on an initial set of 10 interviews and progressed through the six phases. Each interview was transcribed by the first author (removed for blinding) and then read through several times while patterns in the data and initial ideas for coding were noted. Following this, the interview data were systematically coded by organising data extracts into meaningful groups. The first author then further refined these codes, collapsing some into more inclusive codes, and removing some. These codes and their corresponding extracts were reviewed independently by the first (removed for blinding) and third author (removed for blinding), and analysed to organise them into themes and sub themes. These authors then reconvened to discuss and contrast their thematic groupings, a consensus was reached, and an initial thematic map was constructed. The collated coded extracts were then reviewed again independently by both of these authors for internal homogeneity and external heterogeneity in relation to the themes (Patton, 1990). The authors then reviewed and contrasted their themes and collaboratively nominated finalised themes. These were then considered in relation to the data set more broadly through re-reading of the interview transcripts. The themes were then defined and refined to generate thematic maps (depicted in Figures 1 and 2), and interpreted for the analysis. Following this, a further three participants were interviewed and data was transcribed and analysed using the themes and sub-themes already identified to verify their reliability, as well as concurrently attempting to identify any new themes or contradictory data. Although some contradictory views were observed in the data (and have been integrated into the results below), no new relevant themes arose, and the views 
expressed were essentially consistent with the initial pool of 10 interviews. Given this, it was determined that data saturation had occurred. The sample size was congruent with expectations of data saturation in a purposive and homogenous sample such as this (Francis et al., 2010; Guest, Bunce, \& Johnson, 2006).

\section{Results}

The analysis produced themes relating to general perceptions of the intervention, including a sub-theme of worksheets, and change processes with associated sub-themes. The percentage of interviewees independently expressing these ideas is shown in Table 1.

\section{Experience of the Intervention}

All participants reported their experience with CRT to be a positive and helpful one.

Feeling better and less depressed. The majority of participants $(n=10,77 \%)$ also referred to reductions in depressive symptoms, such as improved mood or less negative thoughts, and reported feeling better and less depressed as a result of the therapy.

"Generally it was very positive. I think overall it was quite positive, the outcome of it, and I definitely feel like it has helped having it. I'm glad that I did it" - 20 year-old male.

Acceptability. Almost all of the participants $(n=11,85 \%)$ found the predominant focus of talking about the past to be acceptable. Many discussed how experiences from their past were relevant for the problems they were currently facing, 
and there was utility in exploring their life through reminiscence. This was true even for participants for whom significant improvements in depressive symptoms were not observed.

"I did like that approach, rather than just talking about stuff that is happening now. It did help”.

"It was a new technique that I've never actually thought about, that you can think about the past to fix your future kind of thing. I really liked it" 22 year-old female.

"I think it was useful, especially for me, it was weird because it was really relevant to how Ifeel in the world... It was pretty relevant, in a surprising way, and I can see why it is a helpful method of counselling” - 23 year-old female.

More flexibility needed in the approach. Despite the generally positive reception, concern was voiced by over half of the participants $(n=7,54 \%)$, who perceived a need for more flexibility in the intervention. At times they felt that they were not able to talk about what they wanted to due to the structure of the therapy and the assigned topics each week. One participant suggested that having a week specifically on friends and romantic relationships could be helpful. One participant reported wanting to 
focus more on the present, while five participants commented that some reminiscence content was not relevant for them at that time.

"I think it would have been good to say that isn't really relevant to now, so we can focus on what's happening now instead of that" - 21 year-old male.

Good having someone to listen. The majority of the participants $n=9,(69 \%)$ reported that having someone to listen and to share their thoughts, feelings, and memories with was beneficial. Participants commented on how understanding was communicated through common factors of empathy, unconditional positive regard, and warmth.

Some memories difficult or confronting to discuss. Some participants $(n=7$, $54 \%$ ) reported finding it difficult to remember and express how they had felt in the past, or confronting to discuss upsetting memories. However, this was not typically discussed as an insurmountable barrier to engaging in reminiscence, but rather a part of the process of change.

“[it was]... a little bit emotional just having to kind of go through them again. Like remember them and then talk through them. Some past experiences it was a bit hard to remember certain things, but talking through them just generally and then more in-depth was good" - 21 yearold female. 
Good length/but option for more sessions if needed. Over half of participants $(n=7,54 \%)$ reported that six sessions was an adequate amount of therapy with this approach.

"It was good. It was a good amount....Yeah, definitely it was just the right amount" - 21 year-old female.

However, just under half of participants $(n=6,46 \%)$ also believed that more sessions may have been beneficial, with two indicating a desire for additional sessions (one with no symptomatic change and one deteriorated) and four stating that additional sessions were not needed personally, but could be offered as an option. Two participants suggested that a "check-in" or "catch-up" session at a later date would have been useful.

"Yeah, I think that six is a good amount but maybe if there was a buffer zone, if you felt like you needed more then you have an option. Yeah, a check-in session would be good" - 23 year-old female.

Would recommend to others. All participants advised they would recommend CRT to other people. However, three caveats were raised by different participants: it may not be suitable for social anxiety or for older people, it might be better if balanced with more discussion of present issues, and it is more suited to people who are motivated to change. 
Worksheets. The majority of participants $(n=10,77 \%)$ reported finding the homework sheets to be useful primers for discussion the following week, or springboards for discussion during sessions. Participants spoke of how the sheets prompted them to think about the topic to be discussed, facilitated the process of reflection and introspection, and increased their awareness of related memories from their life.

"Like, the week before I could use it to check around the back of my mind, what I feel about my family or what my kind of feelings are towards this aspect of my life... I found that the worksheet, a little bit of mental preparation, was really beneficial" - 21 year-old female.

Ideas sparked by these worksheets were viewed as a starting point for exploration of the topic. However, over half of the participants $(n=7,54 \%)$ found the worksheets difficult to complete between sessions, citing reasons of forgetfulness, procrastination, finding it hard to make the time, and being unable to think of answers. Although the majority of participants $(n=10,77 \%)$ expressed their appreciation of the role of the questions in stimulating reminiscence, some thought about them informally rather than completing them in a written format before sessions as prescribed.

\section{Change Processes}

\section{Cognitive reframing.}

Reflection and perspective. Almost all the participants $(n=11,85 \%)$ discussed how recounting and reviewing memories through the intervention helped them to generate new perspectives on events in their lives. They spoke of how it fostered more 
adaptive interpretation of these experiences and associated thoughts about themselves.

Reflecting on the past in greater detail, and Socratic questioning by the therapist to elicit different perspectives were reported as being valued by the participants.

"There were a lot of things that we talked about that I never really thought about in the way that we ended up looking at them. And I think that was really helpful" - 20 year-old male.

Developing a balanced and helpful interpretation of experiences. Almost all of the participants $(n=11,85 \%)$ reported developing a more balanced and helpful interpretation of past events and experiences through the processes of guided reminiscence. This involved taking a more even-handed and rational approach to understanding themselves and their lives with less focus on negatives, an increased saliency of positive experiences, and seeing useful or positive aspects in otherwise negative experiences.

'[I'm] definitely thinking about things differently, I'd say that after this six weeks, or however many it's been, I definitely think differently about some past events and kind of have reframed them in my mind" - 19 year-old male.

"Basically just every time we looked at kind of the aspects of myself or of my past that I wasn't that happy with I would usually always come to the conclusion that I was being too hard on myself and that I'd actually been 
COGNITIVE-REMINISCENCE THERAPY

doing better with that kind of specific issue than I thought" - 22 year-old

male.

Less ruminating and gaining closure. A majority of the participants $(n=9,69 \%)$ reported that this process led to less ruminating on negative experiences, and that they were able to work through feelings of guilt, self-blame, regret, or failure, and make peace or gain closure with issues from their past that had been troubling them.

"Just talking about it kind of lifted a weight off my chest and he helped me to see that it wasn't really a thing to feel guilty about and there's other ways of looking at it" - 21 year-old female.

"I think the sessions have helped me to see my life for the good things and the bad things. I feel that from speaking on what my life is like I've been able to let go of some guilt that I've been harbouring”- 21 year-old female.

\section{Awareness of a life story.}

Continuity in the life narrative. Becoming more aware of continuity and cohesion between experiences across the lifetime as a result of reminiscence was a common theme among participants $(n=9,69 \%)$. Many referred to having an increased awareness and insight into the reasons why things had happened in their life and the effects of certain experiences on the course of their life. The participants reported seeing connections between circumstances in the past and the present, and finding it a positive experience to 
COGNITIVE-REMINISCENCE THERAPY

observe a path in their life, or feel a sense of connection with an emerging narrative identity. Participants referred to specific CRT techniques of outlining the narrative of experiences, questioning why they might be important, and collaboratively abstracting instances to reflect a broader theme about them and their life.

"The reason I started was that I was kind of lost...after the sessions I reckon I've got a bit of guide now, and I've got a path that I'm following" -22 year-old female.

"It may not be that clear, but I had taken a general direction [in life]. It was just really nice to see it that way" - 21 year-old male.

Feeling hopeful about the future. The majority of participants $(n=9,69 \%)$ reported that as they abstracted experiences in their life to create a sense of continuity they felt more hopeful about the future than they had previously been, which in turn helped to lift their depression. This included remarks about how they now had a more positive outlook for their life, felt more optimistic, more strongly identified with particular directions to move their life in, or had a heightened awareness that there were a range of possibilities for their future.

"I'm pretty optimistic, I mean compared to before. ... whereas before I was really scared by not knowing what I was going to do now it's kind of being happy that there's so many options open. And that's the thing, that I didn't 
think there was that many options before whereas there are. And yeah, I guess it's good that I can see a kind of future, and I'm not upset" - 21 yearold female.

\section{Learning from the past to cope with challenges.}

Feeling more confident. The majority of participants $(n=10,77 \%)$ reported that instrumental reminiscence was an important factor in feeling more self-efficacious, and subsequently less depressed. A generalised sense of confidence and capability was perceived as arising from the therapist taking time to highlight and emphasise previous successful coping experiences. Participants noted that exploring the ways they had managed to overcome adversity or face problems helped create a view of oneself as being able to face issues or challenges against a background of competence.

"So I'm looking at things, how I'm able to cope with situations or whether I've achieved things in life, yes, it's made me look at myself in a way that I think "okay, I'm able to do that" - 18 year-old female.

Gaining tools to help with coping. In addition to feeling more confident, almost all participants $(n=12,92 \%)$ reported feeling as though they had gained some psychological or behavioural tools for coping with challenges and overcoming adversity. Whilst some of these tools came directly from the structured problem-solving framework that was used, most were identified as methods or strategies from past instances of coping 
that could be drawn on again. Participants commented explicitly that they felt they were now better equipped to deal with problems into the longer-term future.

"It made me realise that yes, in the past I have been able to deal with them, so I should use those same methods, those same things that I used then" - 18 year-old female.

One participant reported that he felt generally better equipped to cope with stressors, but had not gained any further specific strategies for current problems.

Increased self-worth and self-acceptance. Most participants $(n=10,77 \%)$ reported having a more positive evaluation of themselves in emotional and attitudinal terms, and a stronger feeling of self-worth through guided reminiscence. The process of focussing on achievements across their lifetime and experiences had made them proud of themselves, have more trust and conviction in decisions they have made in the past, and believe in themselves, leading to stronger self-esteem and less depression. Some participants also referred to having more self-compassion, and being more reliant on an internally-derived standard of worth as opposed to perceived external standards.

"I think that I value myself a little bit more now. Having sort of talked about some of the good things that I've managed to achieve, and the good relationships I've held onto and developed over the years, and how I've sort of grown as a person, has given me a bit more self-assuredness" - 21 yearold female. 


\author{
"I look at myself a lot differently, I'm kind of seeing myself more positively \\ and [I'm] focussing on my positive attributes and not focussing on the \\ negatives" - 19 year-old male.
}

One participant reported that she had not changed in terms of her self-regard, one conveyed that her self-esteem had not changed as a result of receiving the therapy, and one was ambivalent about whether change had occurred yet, but felt that this would happen over time. All three of these participants reported feeling more confident about themselves and dwelling less on negative events from their past.

\title{
Discussion
}

This study represents the first qualitative research into young adults' experience of reminiscence-based therapy for depressive symptoms, and specifically on CRT. Reminiscence-based therapies have rarely been evaluated with age groups outside of older adulthood (Pinquart \& Forstmeier, 2012). In addition to the reductions in depressive symptoms reported in the RCT, it is noteworthy that the young adults found the approach acceptable and helpful. It is encouraging that individuals of this age, despite typically being more future-oriented than older adults (Carstensen, 2006), found it to be a positive experience. This lends credence to the notion that this type of therapeutic approach is acceptable for individuals across the adult lifespan (removed for blinding).

The common therapeutic factor of having someone who listened was a prevalent theme related to positive change across interviews. This supports the notion that the therapeutic relationship is perceived to promote change in CRT, as is common to 
psychotherapies in general (Lambert \& Barley, 2001). Other perceived processes of change more specific to guided reminiscence in this intervention were noted, and consistent with the integrative nature of CRT techniques. The theme and subthemes of generating new perspectives through cognitive reframing reflect the cognitive therapy and narrative techniques used in CRT, whereby individuals are encouraged to use reasoning to evaluate their experiences in more rational, balanced and adaptive ways. CRT focuses on past experiences rather than the predominantly (but not exclusively) present temporal focus of cognitive therapy (Beck et al., 1979). Nonetheless, the cognitive therapy techniques of identifying and restructuring unhelpful beliefs and attenuating negativistic appraisals that were used to review experiences and reconstruct narratives were perceived by individuals as helping them to take develop more adaptive perspectives. A key aspect of integrative reminiscence is reconciling negative experiences, which was also identified by the participants as occurring in therapy. The participants also perceived that therapy content involved in instrumental reminiscence (not referred to by them directly) led to increases in confidence and a sense of having tools with which to cope with challenges. This is congruent with one aim of CRT, explained clearly at the outset of therapy, whereby therapists attempt to highlight how individuals constructively approached previous challenging situations, promoting their achievements and highlighting the steps they took.

In the narrative tradition implicit in many reminiscence-based therapies, the experiences that individuals discuss are integrated and abstracted to higher-order narratives about the self that balance positive and negatively-valenced experiences and imbue them with a sense of continuity and existential meaning. Indeed, participants did 
identify this process as being a helpful part of CRT. Identification of the role of the life story, becoming aware of relatedness between occurrences in the past and present and having these more cohesively integrated were discussed as leading to a more positive future-orientation. These findings are largely supportive of previous quantitative studies (e.g. Cappeliez \& Robitaille, 2010; removed for blinding) and theoretical propositions (Watt \& Cappeliez, 1995) that indicate adaptive reminiscence is associated with increased self-esteem, self-efficacy and perceived control, and optimism.

Contrary to previous findings (e.g., Binder et al., 2009; Westerhof, Bohlmeijer, van Beljouw, \& Pot, 2010; Xiao et al., 2012), perception of meaning in life was not a theme explicitly identified by participants. Prior studies, however, have been conducted with older adults who inherently have a vastly larger number of years over which to identify substantial and meaningful experiences in their life, and are more likely to have established fruitful work or other occupational histories, longer-lasting relationships, and achieved or attempted important psychosocial goals such as generativity. Further, older adults may be more inclined to utilize this experience to establish a sense of their past as having been purposeful, given their increased awareness of less time left ahead in life (Carstenson, 2006). The young adults did not explicitly refer to meaning in life, but instead spoke of an increased awareness of emerging life narratives and connectedness between experiences. It has been argued that this sense of narrative identity represents a component of well-being that provides psychosocial integration of meaning and frames transformative experiences as being purposeful (Bauer, McAdams, \& Pals, 2008). This may represent an alternative expression of the general concept of meaning in life. Further, individuals in this stage of emerging adulthood may perceive purpose or 
meaning in life in this therapy to be related to longer-term goals that are still being formed, rather than meaning as embedded in the past.

Regarding criticisms of the intervention, one participant voiced the desire to focus more on current issues, and other participants suggested a less restrictive structuring of topics. This may have been due to variance between individuals in how relevant they perceived particular topics of reminiscence to be in relation to causes of their depression (e.g. interpersonal conflict, perceived inability to cope). Offering choices in how reminiscence topics are covered, or offering a broader choice of topics, such as social relationships, may help address this. These may represent particular needs in this population, and aid in increasing its acceptability without compromising treatment integrity. In CRT, a brief problem-solving component serves to promote agency to address current problems, whilst the building of narratives about the self through reminiscence also leads on to views of the present and future self. It is difficult to conceive of how to incorporate more work on present issues without compromising the focus on reminiscence and narrative that typifies this intervention. Interestingly, participants did not distinguish the active problem-solving component of the therapy as being strongly related to change relative to other processes. This may reflect the fact that only a small amount of time was spent on the problem-solving activity, or that other aspects of the intervention were viewed as being more helpful.

Participants' comments on the desire for additional sessions or a booster session suggest some flexibility in treatment length may be advantageous. In Watt and Cappeliez's (2000) trial of their integrative and instrumental reminiscence-based interventions, on which this therapy was modelled, such booster sessions were provided. 
These may serve to provide individuals with an opportunity to check-in regarding ongoing use of reminiscence or debrief on how functional or psychological changes have progressed. Given the time-limited nature of CRT, offering a booster session at a negotiated interval after the sixth session (e.g. one or three months later) may be received favourably by some individuals, and provide the sense of ongoing support following the initial course of sessions. Participants found homework sheets useful, but difficult to formally complete between sessions. These barriers did not appear to be insurmountable though (e.g., forgetting), nor underpinned by negative attitudes towards the worksheets. Given the effects of homework on outcomes in psychotherapy (e.g. Kazantzis, Whittington, \& Dattilio, 2010), placing a stronger emphasis on their function and more focused implementation, perhaps involving collaboratively nominating a time for completion during the week, may be useful to increase participants' willingness and compliance.

Although the sample size in this study was small, it was homogenous in terms of age and presenting issue, and all individuals received the same manualised intervention. The high prevalence of the themes across the sample also supports their credibility as "common" perceptions, as well as lending credence to their importance. Given these considerations, the findings in this study may have some transferability to other young adults who receive CRT. A larger sample size would be of value in future studies of this nature though, in order to increase confidence that these themes are representative of young adults' views of CRT. It might also increase the chance of identifying exceptions to them or additional themes, and unearth other potential ways to refine and improve this therapy. Validity checks of the analyses by independent researchers may have been 
useful. Although the third author satisfied this role to some degree, this likely did not fully mitigate biases that may have arose. Social desirability bias is also a possibility in this study. The participants may have felt obliged to provide a positive impression of the intervention, possibly to satisfy the researchers and/or create an impression of being amicable and appreciative to the interviewer. We did try to mitigate this by using an interviewer who was not otherwise involved in the therapy, probed for any negative or critical opinions of the interviewees, and conveyed that their interviews would be transcribed and de-identified for analysis. The young adults did provide some clear criticisms of the therapy, indicating that social desirability, if present, only partially influenced their responses. We might have included quantitative items relating to perceptions of the intervention, as the anonymity may have reduced any bias. While this would not capture the detail in responses that an interview approach does, it may have been a useful adjunct. However, we suspect that for the reasons stated it is a difficult task to fully mitigate social desirability effects in a study where a treatment involving personal interaction such as this is provided. Other limitations are that participants were helpseeking for depressive symptoms, but not formally diagnosed. We also did not assess the duration of participants' depressive symptoms, and so it is unknown whether these findings would be relevant for more chronic episodes of depression, or treatment-resistant depression. Future research may seek to examine whether young adults' perceptions differ comparatively in regards to reminiscence-based therapies that utilize a different framework for treatment, or adopt an informal, less structured or unguided approach to reminiscence. 
Overall, these findings indicate that CRT may be acceptable and helpful for young adults in emerging adulthood. In the context of a critical period in life of psychosocial development (e.g. the transition from adolescence to adulthood, increased autonomy, the development of social, occupational, vocational roles etc.), the young adults viewed the intervention as providing a forum to adaptively explore and learn from their past, and utilized this understanding to strengthen their self-concept, narrative identity, perceived control and ability to cope. In practice, CRT might be particularly suitable for young adults with existential anxiety, or who are in the midst of significant disruptions to their identity and/or are actively exploring alternative self-identities when transitioning into different roles or circumstances. Young adults who signal a desire for self-reflection on their life might also be a good fit for this approach. Given that CRT content is based primarily on the participant's narratives, and is not heavily skills-based, it may be an approach that can accommodate for a range of philosophical views regarding personal identity and the relation of the self to others and the world. For example, perspectives based on individualism, free will, and self-determination of identity, or more collectivist or social-constructionist views of meaning and identity. However, CRT still may not suit individuals that prefer a more directive, and blatantly technique-based approach. On these points, and its effectiveness in relation to other therapies, further research into CRT is needed. 
References

Arnett, J. J. (2004). Emerging adulthood: The winding road from late teens through the twenties. New York: Oxford University Press.

Beck, A. T., Rush, A. J., Shaw, B. F., \& Emery, G. (1979). Cognitive therapy of depression. New York, NY: Guildford Press.

Billings, A. G., \& Moos, R. H. (1982). Psychosocial theory and research on depression: An integrative framework and review. Clinical Psychology Review, 2, 213-237. doi:10.1016/0272-7358(82)90013-7

Binder, B. K., Mastel-Smith, B., Hersch, G., Symes, L., Malecha, A., \& McFarlane, J. (2009). Community-dwelling, older women's perspectives on Therapeutic Life Review: a qualitative analysis. Issues in Mental Health Nursing, 30, 288-294. doi:10.1080/01612840902753885

Bluck, S., \& Alea, N. (2008). Remembering being me: The self continuity function of autobiographical memory in younger and older adults. In F. Sani (Ed.), Self Continuity: Individual and Collective Perspectives (55-70). New York: Psychology Press.

Bluck, S., \& Alea, N. (2009). Thinking and talking about the past: Why remember? Applied Cognitive Psychology, 23, 1089-1104. doi:10.1002/acp.1612.

Braun, V., \& Clarke, V. (2006). Using thematic analysis in psychology. Qualitative research in psychology, 3(2), 77-101. doi:10.1191/1478088706qp063oa

Cachia, M., \& Millward, L. (2011). The telephone medium and semi-structured interviews: a complementary fit. Qualitative Research in Organizations and 
Management: An International Journal, 6, 265-277.

doi:10.1108/17465641111188420

Cappeliez, P. (2002). Cognitive-reminiscence therapy for depressed older adults in day hospital and long-term care. In Webster, J. D. and Haight, B. K. (eds), Critical Advances in Reminiscence Work: From Theory to Application. Springer, New York, $300-313$.

Cappeliez, P., \& Robitaille, A. (2010). Coping mediates the relationships between reminiscence and psychological well-being amongst older adults. Aging and Mental Health, 14, 807-818. doi:10.1080/13607861003713307

Carstensen, L. L. (2006). The influence of a sense of time on human development. Science, 312, 1913-1915. doi:10.1126/science.1127488

Coryell, W., Solomon, D., Leon, A., Fiedorowicz, J. G., Schettler, P., Judd, L., \& Keller, M. (2009). Does major depressive disorder change with age?. Psychological Medicine, 39, 1689-1695. doi:10.1017/S0033291709005364

Francis, J. J., Johnston, M., Robertson, C., Glidewell, L., Entwistle, V., Eccles, M. P., \& Grimshaw, J. M. (2010). What is an adequate sample size? Operationalising data saturation for theory-based interview studies. Psychology and Health, 25, 12291245. doi:10.1080/08870440903194015

Guest, G., Bunce, A., \& Johnson, L. (2006). How many interviews are enough? An experiment with data saturation and variability. Field methods, 18(1), 59-82. doi:10.1177/1525822X05279903 
Habermas, T., \& Bluck, S. (2000). Getting a life: The emergence of the life story in adolescence. Psychological Bulletin, 126, 748-769. doi:10.1037OT0332909.126.5.741

Hallford, D., \& Mellor, D. (2013). Reminiscence-based therapies for depression: Should they be used only with older adults? Clinical Psychology: Science and Practice, 20, 452-468. doi:10.1111/ cpsp.12043

Hallford, D. J., Mellor, D., \& Cummins, R. A. (2013). Adaptive auto- biographical memory in younger and older adults: The indirect association of integrative and instrumental reminiscence with depressive symptoms. Memory, 21, 444-457. doi:10.1080/ 09658211.2012.736523

Hallford, D. J., \& Mellor, D. (2016a). Autobiographical memory and depression: Identity-continuity and problem-solving functions indirectly predict symptoms over time through psychological well-being. Applied Cognitive Psychology, 30, 152-159. doi:10. 1002/acp.3169

Hallford, D. J., \& Mellor, D. (2016b). Autobiographical memory- based intervention for depressive symptoms in young adults: A randomized controlled trial of cognitivereminiscence therapy. Psychotherapy and Psychosomatics, 85, 246-249. doi:10.1159/ 000444417

Hallford, D. J., \& Mellor, D. (2016c). Brief reminiscence activities improve state wellbeing and self-concept in young adults: A ran- domised controlled experiment. Memory, 24, 1311-1320. doi:10. 1080/09658211.2015.1103875 
Hasin, D. S., Goodwin, R. D., Stinson, F. S., \& Grant, B. F. (2005). Epidemiology of major depressive disorder: results from the National Epidemiologic Survey on Alcoholism and Related Conditions. Archives of General Psychiatry, 62, 10971106. doi:10.1001/archpsyc.62.10.1097

Issakainen, M., \& Hänninen, V. (2016). Young people's narratives of depression. Journal of Youth Studies, 19(2), 237-250. doi:10.1080/13676261.2015.1059927.

Jacobson, N. S., \& Truax, P. (1991). Clinical significance: a statistical approach to defining meaningful change in psychotherapy research. Journal of Consulting and Clinical Psychology, 59(1), 12-19. doi:10.1037/0022-006X.59.1.12

Lambert, M. J., \& Barley, D. E. (2001). Research summary on the therapeutic relationship and psychotherapy outcome. Psychotherapy: Theory, Research, Practice, Training, 38(4), 357-361. doi:10.1037/0033-3204.38.4.357

Lovibond, P. F., \& Lovibond, S. H. (1995). Manual for the Depression Anxiety Stress Scales, (2 ${ }^{\text {nd }}$ ed.). Sydney, Australia: Psychology Foundation of Australia.

Karimi, H., Dolatshahee, B., Momeni, K., Khodabakhasi, A., Rezaei, M., \& Kamrani, A. A. (2010). Effectiveness of integrative and instrumental reminiscence therapies on depressive symptom reduction in institutionalised older adults: An empirical study. Aging and Mental Health, 14(7), 881-887. doi:10.1080/13607861003801037

Korten, N., Comijs, H. C., Lamers, F., \& Penninx, B. W. (2012). Early and late onset depression in young and middle aged adults: Differential symptomatology, characteristics and risk factors? Journal of Affective Disorders, 138, 259-267. doi:10.1016/j.jad.2012.01.042.

Kazantzis, N., Whittington, C., \& Dattilio, F. (2010). Meta - Analysis of Homework Effects in Cognitive and Behavioral Therapy: A Replication and Extension. Clinical 
Psychology: Science and Practice, 17(2), 144-156. doi:10.1111/j.1468-

2850.2010.01204.x

Korte, J., Westerhof, G. J., \& Bohlmeijer, E. T. (2012). Mediating processes in an effective life-review intervention. Psychology and Aging, 27, 1172-1181. doi: $10.1037 / \mathrm{a} 0029273$

Malette, J., \& Oliver, L. (2006). Retirement and existential meaning in the older adult: A qualitative study using life review. Counselling, Psychotherapy, and Health, 2(1), 30-49. Retrieved from https://www.mentalhealthacademy.net/journal_archive/ cph0620.pdf

May, K., Strauss, C., Coyle, A., \& Hayward, M. (2012). Person-based cognitive therapy groups for distressing voices: a thematic analysis of participant experiences of the therapy. Psychosis: Psychological, Social, and Integrative Approaches, 6(1), 16-26. doi:10.1080/17522439.2012.708775

McAdams, D. P. (2001). The psychology of life stories. Review of General Psychology, 5(2), 100-122. doi:10.1037/1089-2680.5.2.100

McDougall, G. J., Blixen, C. E., \& Suen, L. J. (1997). The process and outcome of life review psychotherapy with depressed homebound older adults. Nursing Research, 46, 277-283. doi:10.1097/00006199-199709000-00006

Nezu, A. M. (2004). Problem-solving and behaviour therapy revisited. Behavior Therapy, 35, 1-33. doi:10.1016/S0005-7894(04)80002-9

Novick, G. (2008). Is there a bias against telephone interviews in qualitative research?. Research in Nursing \& Health, 31, 391-398. doi:10.1002/nur.20259 
Patton, M.Q. (1990). Qualitative evaluation and research methods ( $2^{\text {nd }}$ ed.). Thousand Oaks, U.S.: Sage Publications, Inc.

Pinquart, M., Forstmeier, S. (2012). Effects of reminiscence interventions on psychosocial outcomes: A meta-analysis. Aging and Mental Health, 16, 541-558. doi:10.1080/13607863.2011.651434

Purcell, R., Ryan, S., Scanlan, F., Morgan, A., Callahan, P., Allen, N. B., \& Jorm, A. F. (2013). A guide to what works for depression in young people (2nd edition). Melbourne, Australia: Beyondblue.

Rohde, P., Lewinsohn, P. M., Klein, D. N., Seeley, J. R., \& Gau, J. M. (2013). Key characteristics of major depressive disorder occurring in childhood, adolescence, emerging adulthood, and adulthood. Clinical Psychological Science, 1(1), 41-53. doi:10.1177/2167702612457599

van Rooij, A. J., Zinn, M. F., Schoenmakers, T. M., \& van de Mheen, D. (2012). Treating internet addiction with cognitive-behavioral therapy: a thematic analysis of the experiences of therapists. International Journal of Mental Health and Addiction, 10(1), 69-82. doi:10.1007/s11469-010-9295-0

Singer, J. A., Blagov, P., Berry, M., \& Oost, K. M. (2013). Self-defining memories, scripts, and the life story: Narrative identity in personality and psychotherapy. Journal of Personality. doi:10.1111/jopy.12005

Shellman, J., Mokel, M., \& Hewitt, N. (2009). The effects of integrative reminiscence on depressive symptoms in older African-Americans. Western Journal of Nursing Research, 31(6), 772-786. doi:10.1177/0193945909335863

Slade, T., Johnston, A., Browne, M. A. O., Andrews, G., \& Whiteford, H. (2009). 2007 National Survey of Mental Health and Wellbeing: Methods and key findings. 
Australian and New Zealand Journal of Psychiatry, 43, 594-605. doi:10.1080/ 00048670902970882

Watt, L. M., \& Cappeliez, P. (1995). Reminiscence interventions for the treatment of depression in older adults. In B.K. Haight \& J. D. Webster (Eds.), The Art and Science of Reminiscing: Theory, Methods, and Applications (pp. 221-232).

Washington, DC: Taylor and Francais.

Watt. L. M., \& Cappeliez, P. (2000). Integrative and instrumental reminiscence therapies for depression in older adults. Intervention strategies and treatment effectiveness. Aging and Mental Health, 4, 166-177. doi:10.1080/13607860050008691

Westerhof, J. D., Bohlmeijer, E. T., van Beljouw, I. M. J., \& Pot, A. M. (2010). Improvement in personal meaning mediates the effects of a life review intervention on depressive symptoms in a randomised controlled trial. The Gerontologist, 50, 541-549. doi:10.1093/geront/gnp168

Wong, P. T. P. (1995). The processes of adaptive reminiscence. In B. K. Haight \& J.D. Webster (Eds.). The art and science of reminiscing: Theory, methods, and applications (pp. 23-35). Washington, DC: Taylor and Francais.

Xiao, H., Kwong, E., Pang, S., \& Mok, E. (2012). Perceptions of a life review programme among Chinese patients with advanced cancer. Journal of Clinical Nursing, 21, 564-572. doi:10.1111/j.1365-2702.2011.03842.x

Zisook, S., Lesser, I., Stewart, J. W., Wisniewski, S. R., Balasubramani, G. K., Fava, M., . . Rush, A. J. (2007). Effect of age at onset on the course of major depressive disorder. American Journal of Psychiatry, 164, 1539-1546. doi:10.1176/appi.ajp. 2007.06101757 

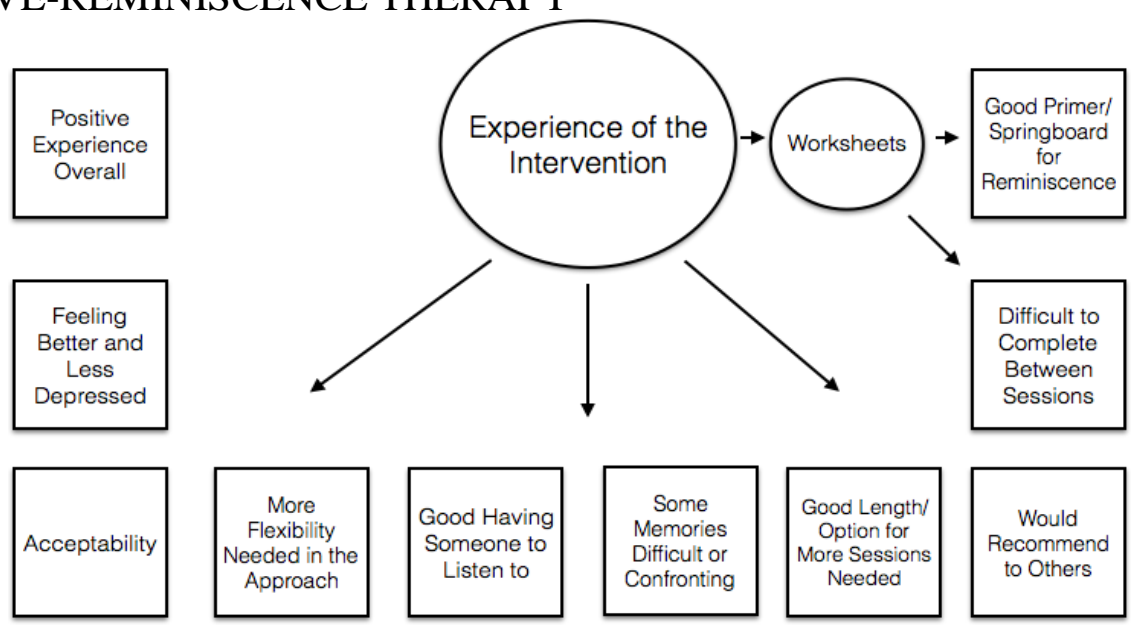

Figure 1. Final thematic map of the Experience of the Intervention theme and sub-themes 


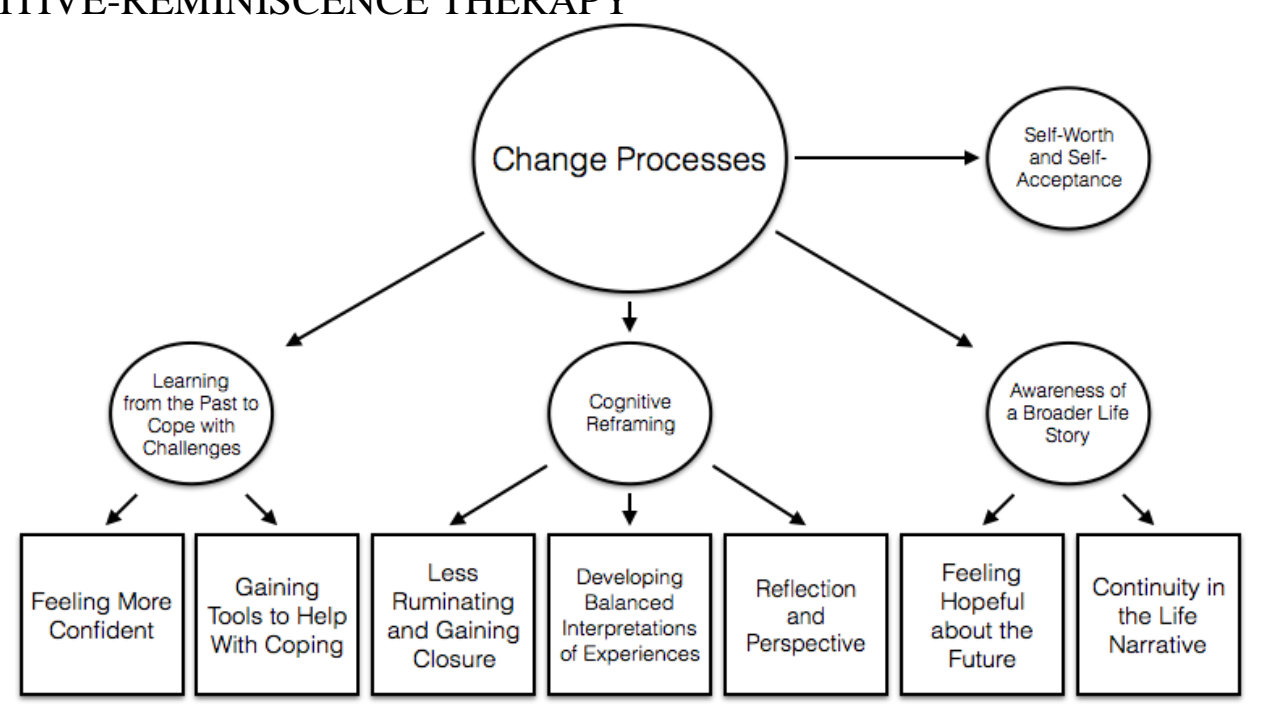

Figure 2. Final thematic map of the Change Processes theme and sub-themes 
Table 1.

Themes and Sub-themes Identified from the Interview Data Set and the Percentage of Individuals who Expressed These Ideas in Interviews

$\%$ of

Themes and Sub-themes

Individuals

Experience of the Intervention

Positive experience overall

Feeling better and less depressed

Acceptability

More flexibility needed in the approach

Good having someone to listen

Some memories difficult or confronting to discuss

Good length/but option for more sessions if needed

Would recommend to others

Worksheets

Good primer/springboard for reminiscence

Difficult to complete between sessions

Cognitive Reframing

Reflection and perspective

Developing a balanced and helpful interpretation of experiences

Less ruminating and gaining closure

Awareness of a Life Story

Continuity in the life narrative

Feeling hopeful about the future

Learning from the Past to Cope with Challenges

Feeling more confident

Gaining tools to help with coping 
